\title{
Predictors of Acne Vulgaris among Adolescents in Kuwait
}

\author{
Mona AlKhabbaz ${ }^{a}$ Abdullah Al-Taiar ${ }^{b}$ Marwa Saeed ${ }^{c}$ Reem Al-Sabah ${ }^{b}$ \\ Ahmed N. Albatineh ${ }^{b}$ \\ ${ }^{a}$ Quality and Accreditation Directorate, Ministry of Health, Kuwait, Kuwait; ${ }^{b}$ School of Community and \\ Environmental Health, College of Health Sciences, Old Dominion University, Norfolk, VA, USA; ${ }^{\mathrm{C} M u b a r a k}$ Alkabeer \\ Polyclinic, Ahmadi Health Area, Kuwait, Kuwait
}

\section{Significance of the Study}

- This study reports the prevalence of acne vulgaris in a nationally representative sample of adolescents in Kuwait.

- The study also investigated the link between acne vulgaris and various factors including diet, obesity/ overweight, and various laboratory measurements such as vitamin $\mathrm{B}_{12}$.

\section{Keywords}

Acne vulgaris · Predictors · Adolescents · Kuwait

\begin{abstract}
Objectives: The prevalence of acne vulgaris (AV) among adolescents in Kuwait has remained mostly unknown. This study aimed to estimate the prevalence of $A V$ and its predictors among adolescents in Kuwait. Subjects and Methods: A cross-sectional study was conducted on 714 participants who were randomly selected from public schools using multistage cluster random sampling. The presence or absence of $A V$, as well as severity of AV, were assessed using the Global Acne Grading Scale. Data on risk factors and potential confounders were collected from the parents through a self-administered questionnaire, and from the adolescents by faceto-face interview. Weight and height were measured in a standardized manner. Results: Of 714 participants, 320 (44.8\%; 95\% Cl 41.1-48.6\%) self-reported AV. By clinical ex-
\end{abstract}

amination, AV was found among 479 (67.1\%; 95\% Cl 63.5$70.5 \%$ ) participants. Factors that were found to be significantly associated with AV in multivariable analysis were age, gender, nationality, and BMI categories. Females compared to males (adjusted prevalence ratio [APR] 1.16 [95\% Cl 1.05$1.25] ; p=0.006$ ) and overweight or obese adolescents compared to normal weight (APR 1.15 [95\% Cl 1.02-1.26] and 1.14 [95\% Cl 1.02-1.23], respectively; $p=0.026$ ) were more likely to have AV. None of the food items studied showed an association with AV in multivariable analysis. Conclusion: The prevalence of self-reported AV seems to be lower than that reported in other settings, while the prevalence of AV by clinical examination resembles that reported in many other countries. BMI was the only modifiable risk factor associated with AV in multivariable analysis, and none of the food items studied was related to the presence or absence of $\mathrm{AV}$ in multivariable analysis.

(c) 2019 The Author(s) Published by S. Karger AG, Basel

\begin{tabular}{ll}
\hline KARGER & $\begin{array}{l}\text { () } 2019 \text { The Author(s) } \\
\text { Published by S. Karger AG, Basel Oparger }\end{array}$ \\
karger@karger.com & Then access \\
www.karger.com/mpp & $\begin{array}{l}\text { Attribution-NonComm Access article licensed under the Creative Commons } \\
\text { (http://www.karger.com/Services International License (CC BY-NC) } \\
\text { the online version of the article only. Usage and distribution for com- } \\
\text { mercial purposes requires written permission. }\end{array}$
\end{tabular}

Dr. Abdullah Al-Taiar

School of Community and Environmental Health

College of Health Sciences, Old Dominion University

Norfolk, VA 23529 (USA)

E-Mail aaltaiar@odu.edu 


\section{Introduction}

Acne vulgaris (AV) is a common chronic inflammatory condition of pilosebaceous glands. Approximately, $9.4 \%$ of the world's population are affected by $\mathrm{AV}$, with the highest prevalence among post-pubescent teenagers [1]. AV is considered the most common skin disease globally [2] and ranked as the 8th most common disease worldwide [3]. Skin diseases are unlikely to be life-threatening conditions when not accompanied by systemic involvement, yet they are the fourth leading cause of disability worldwide [4]. Of all skin diseases, AV has been identified as the second leading cause of disability-adjusted life years after dermatitis (atopic, contact, and seborrheic dermatitis), causing loss of productivity for patients and caregivers that is equal to the productivity cost of skin cancer [4]. Additionally, AV puts a significant burden on healthcare services and may lead to depression and in severe cases to suicidal ideation and suicide attempts $[5,6]$.

Studies have demonstrated substantial differences in the prevalence of $\mathrm{AV}$ among adolescents and young adults between different countries. Although this may reflect a genuine difference between geographical settings, it could be due to methodological differences (e.g., AV by clinical examination vs. self-reported AV) or due to the fact that different studies targeted different study groups (e.g., military recruits vs. students in schools). In Nigeria, $97.1 \%$ of 17 - to 19 -year-old students were diagnosed with AV by clinical examination [7], while in an online survey in 7 European countries, self-reported AV ranged from $42.2 \%$ in Poland to $73.5 \%$ in the Czech and Slovak Republics among adolescents aged 15-24 years [8]. A literature review of 11 cross-sectional studies of different age groups between 6 and 25 years in several South East Asian countries showed AV prevalence between 9.8 and 91.3\% [1]. Regionally, in a cross-sectional study in Iran, AV was diagnosed in $93.3 \%$ of high school students ( $\geq 17$ years old) [9]. A literature review of 6 cross-sectional studies in the Middle East (Saudi Arabia, Pakistan, and Iran) showed AV prevalence between 13 and $93.2 \%$ among children and young adults (6-28 years) [1].

In Kuwait, there is a paucity of data on the prevalence of AV or its associated factors among adolescents or young adults. In a hospital-based case survey of patients in a public pediatric dermatology clinic, AV comprised about $1.42 \%$ of patients (142 out of 10,000 cases) [10]. This is unlikely to reveal the true burden of AV in Kuwait, as we anticipate that most adolescents with AV would seek medical care from private clinics. Furthermore, factors that have been implicated in developing or aggravat- ing AV such as childhood obesity, using sunscreen, and consumption of sugary and fast foods are all on the rise in Kuwait. We have previously demonstrated that AV is not related to levels of vitamin D [11]. In the present study we aimed to estimate the prevalence of AV in Kuwait and to investigate the association between $\mathrm{AV}$ and various factors including obesity and dietary factors.

\section{Subjects and Methods}

This is a cross-sectional study on adolescents (10.3-16.1 years) that were previously recruited in a project funded by Kuwait University. The project aimed to study vitamin $\mathrm{D}$ status among adolescents in Kuwait and the details of this project have been published previously [12]. In brief, a nationally representative sample of students at public schools was selected through multistage cluster random sampling using probability proportional to size sampling method. With this sampling method, schools with large number of students are given higher probability to be selected compared to schools with small number of students. Data were collected from the parents through a self-administered questionnaire. This included data on the level of education of parents and other sociodemographic characteristics. Data were also collected from adolescents by trained dedicated personnel through face-to-face interviews using a structured questionnaire. The questionnaire included questions on physical activity, smoking, and other lifestyle factors. Blood samples were analyzed for various laboratory indicators including hemoglobin, ferritin, transferrin saturation, parathyroid hormone (PTH), 25 -hydroxyvitamin $\mathrm{D}$, total protein, albumin, and vitamin $\mathrm{B}_{12}$ in addition to other laboratory parameters. Laboratory measurements were conducted in an accredited laboratory under strict measures of quality control. Weight was measured to the nearest $0.1 \mathrm{~kg}$ and height to the nearest $0.1 \mathrm{~cm}$ using a digital weight and height scale in a standardized manner. This data collection was performed by the research team of the funded project mentioned above.

Another independent team was formulated to collect data from the same study group on AV and associated factors using face-toface interviews and clinical examination. This was conducted in only a subgroup of 714 adolescents out of 1,416 in the original study (Table 1). We assessed the presence or absence of AV in addition to the severity of AV using the Global Acne Grading Scale (GAGS), which was developed in 1997 by Doshi et al. [13]. GAGS score divides the face, chest, and back into 6 locations (forehead, each cheek, nose, chin, chest, and upper back). The 6 locations are graded separately with each given a factor from 1 to 3 , then the most severe lesion in each area is graded according to a score from 0 to 4 ( 0 = no lesions, $1=$ comedones, $2=$ papules, $3=$ pustules, and $4=$ nodules). The score is multiplied by the factor for all the 6 locations. These scores are added to give the overall GAGS score. The overall score is categorized as follows: "zero" acne is graded as "none," " $1-18$ " is "mild," "19-30" is "moderate," " $31-38$ " is "severe," and " $>39$ " is "very severe." In a subgroup of 186 participants, the diagnosis of AV and grading was conducted by 2 independent physicians. This showed substantial agreement (kappa $60.7 \%$ for presence or absence of acne).

The data on AV were entered into a specifically designed database and linked to the data collected previously using a unique 
Table 1. Sociodemographic characteristics of 1,416 participants in the original study and 714 participants in the current study

\begin{tabular}{|c|c|c|}
\hline Characteristics & $\begin{array}{l}\text { Participants in the } \\
\text { original study }(n=1,416)\end{array}$ & $\begin{array}{l}\text { Participants examined for } \\
\text { acne vulgaris }(n=714)\end{array}$ \\
\hline Age, years & $12.48 \pm 0.94$ & $12.28 \pm 0.81$ \\
\hline \multicolumn{3}{|l|}{ Gender } \\
\hline Male & $694(49.0)$ & $351(49.2)$ \\
\hline \multicolumn{3}{|l|}{ Nationality } \\
\hline Kuwaiti & $1,081(76.3)$ & $511(71.6)$ \\
\hline Non-Kuwaiti & $335(23.7)$ & $203(28.4)$ \\
\hline \multicolumn{3}{|l|}{ Father's education* } \\
\hline No formal education & $15(1.1)$ & $6(0.9)$ \\
\hline Primary/intermediate & $221(16.0)$ & $85(12.1)$ \\
\hline Secondary (high school) & $344(24.9)$ & $174(24.9)$ \\
\hline Diploma & $261(18.9)$ & 139 (19.9) \\
\hline University and above & $542(39.2)$ & $296(42.3)$ \\
\hline \multicolumn{3}{|l|}{ Mother's education* } \\
\hline No formal education & $31(2.2)$ & $12(1.7)$ \\
\hline Primary/intermediate & $152(10.9)$ & $58(8.2)$ \\
\hline Secondary (high school) & $304(21.8)$ & $153(21.7)$ \\
\hline Diploma & $304(21.8)$ & $150(21.3)$ \\
\hline University and above & $605(43.3)$ & $332(47.1)$ \\
\hline \multicolumn{3}{|l|}{ Father's income* } \\
\hline$<500 \mathrm{KWD}$ & $91(6.6)$ & $54(7.8)$ \\
\hline 500-1,000 KWD & $304(22.2)$ & $160(23.2)$ \\
\hline 1,001-1,500 KWD & $421(30.7)$ & $210(30.4)$ \\
\hline $1,501-2,000 \mathrm{KWD}$ & $219(16.0)$ & $102(14.8)$ \\
\hline$>2,000 \mathrm{KWD}$ & $173(12.6)$ & $94(13.6)$ \\
\hline Do not wish to tell & $162(11.8)$ & $71(10.3)$ \\
\hline \multicolumn{3}{|l|}{ Mother's employment status* } \\
\hline Housewife & $488(35.2)$ & $225(32.0)$ \\
\hline Paid employment & $680(49.0)$ & $361(51.4)$ \\
\hline Others & $220(15.8)$ & $117(16.6)$ \\
\hline \multicolumn{3}{|l|}{ Housing* } \\
\hline Rented flat & $510(36.5)$ & $281(39.9)$ \\
\hline Rented house & $163(11.7)$ & $79(11.2)$ \\
\hline Owned flat & $59(4.2)$ & $41(5.8)$ \\
\hline Owned house & $665(47.6)$ & $303(43.0)$ \\
\hline
\end{tabular}

Data presented as mean $\pm \mathrm{SD}$ or $n(\%)$ * Some values are missing.

identification number, and then analyzed using IBM SPSS Statistics for Windows, version 24 (IBM Corp., Armonk, NY, USA) and Stata version 12 (StataCorp., 2011). 95\% CIs for the prevalence of AV were calculated using the exact binomial distribution. Kappa statistic for categorical variables was used to assess the agreement between the 2 clinicians in assessing the diagnosis of AV. BMI was calculated as weight $(\mathrm{kg})$ divided by height $(\mathrm{m})$ squared, and $Z$ score was calculated using the World Health Organization (WHO) growth charts. Overweight was defined as BMI-for-age $>1 \mathrm{SD}$, while obesity as BMI-for-age $>2$ SD. To investigate factors associated with the presence or absence of $\mathrm{AV}$, we used a modified version of logistic regression that calculates prevalence ratio (instead of OR) using the Stata command "oddsrisk." Factors that showed association with AV at $20 \%$ level of significance in univariable analysis (using chi-square test) were considered in multivariable analysis. In univariable and multivariable analysis, only factors associated with AV at $<5 \%$ level of significance using likelihood ratio test were deemed to be statistically significant. The goodness-of-fit of this model was tested using Hosmer-Lemeshow test.

\section{Results}

The mean age was 12.28 (SD 0.81) years and the majority, 511, were Kuwaiti nationals (71.6\%). Table 1 shows the sociodemographic characteristics of 714 participants in this study, in addition to 1,416 who participated in the original study. Of 714 participants, 320 (44.8\%; 95\% CI 
Table 2. Factors associated with clinically examined acne vulgaris among adolescents in Kuwait $(n=714)$ in univariable logistic regression analysis

\begin{tabular}{|c|c|c|c|c|c|}
\hline Variables & $N$ & Prevalence, $n(\%)$ & $\mathrm{PR}^{1}$ & $95 \% \mathrm{CI}$ & $p$ value $^{2}$ \\
\hline Total & 714 & $479(67.1)$ & & & \\
\hline \multicolumn{6}{|l|}{ Age } \\
\hline 10 years & 301 & $189(61.8)$ & 1.00 & Reference & \multirow[t]{3}{*}{0.029} \\
\hline 12 years & 275 & $192(69.8)$ & 1.12 & $1.00-1.21$ & \\
\hline 13 years & 138 & $101(73.2)$ & 1.16 & $1.03-1.27$ & \\
\hline \multicolumn{6}{|l|}{ Gender } \\
\hline Male & 351 & $210(59.8)$ & 1.00 & Reference & \multirow[t]{2}{*}{$<0.001$} \\
\hline Female & 363 & $269(74.1)$ & 1.23 & $1.13-1.33$ & \\
\hline \multicolumn{6}{|l|}{ Nationality } \\
\hline Kuwaiti & 511 & $368(72.0)$ & 1.00 & Reference & \multirow[t]{2}{*}{$<0.001$} \\
\hline Non-Kuwaiti & 203 & $111(54.7)$ & 0.76 & $0.64-0.87$ & \\
\hline \multicolumn{6}{|l|}{ Body mass index categories ${ }^{3}$} \\
\hline Underweight & 11 & $5(45.4)$ & 0.76 & $0.36-1.16$ & \multirow[t]{4}{*}{0.017} \\
\hline Normal weight & 313 & $194(62.0)$ & 1.00 & Reference & \\
\hline Overweight & 158 & $116(73.4)$ & 1.15 & $1.03-1.25$ & \\
\hline Obesity & 232 & $164(70.7)$ & 1.11 & $1.01-1.21$ & \\
\hline \multicolumn{6}{|c|}{ Consumption of low-fat milk in the past 3 months } \\
\hline Never/less than once/week & 470 & $303(64.5)$ & 1.00 & Reference & \multirow[t]{4}{*}{0.028} \\
\hline 1-2 times/week & 98 & $75(76.5)$ & 1.80 & $1.08-2.97$ & \\
\hline 3-5 times/week & 62 & $38(61.3)$ & 0.87 & $0.50-1.50$ & \\
\hline 6 times or more/week & 84 & $63(75.0)$ & 1.65 & $0.97-2.80$ & \\
\hline \multicolumn{6}{|c|}{ Consumption of fruits in the past 3 months } \\
\hline Every day & 171 & $114(66.7)$ & 1.00 & Reference & \multirow[t]{5}{*}{0.014} \\
\hline 4-6 days/week & 76 & $49(64.5)$ & 0.97 & $0.76-1.14$ & \\
\hline 1-3 days/week & 249 & $154(61.8)$ & 0.93 & $0.78-1.06$ & \\
\hline 1 day/week & 153 & $108(70.6)$ & 1.06 & $0.90-1.18$ & \\
\hline Never & 65 & $54(83.1)$ & 1.24 & $1.06-1.35$ & \\
\hline \multicolumn{6}{|c|}{ Consumption of chips/fried food in the past 3 months } \\
\hline Every day & 222 & $135(60.8)$ & 1.00 & Reference & \multirow[t]{5}{*}{0.034} \\
\hline 4-6 days/week & 109 & $69(63.3)$ & 1.03 & $0.87-1.16$ & \\
\hline 1-3 days/week & 220 & $155(70.4)$ & 1.13 & $1.01-1.22$ & \\
\hline 1 day/week & 135 & $102(75.6)$ & 1.19 & $1.06-1.28$ & \\
\hline Never & 28 & $18(64.3)$ & 1.05 & $0.76-1.25$ & \\
\hline \multicolumn{6}{|l|}{ Transferrin saturation } \\
\hline Lower tertile & 234 & $172(73.5)$ & 1.00 & & \multirow[t]{3}{*}{0.008} \\
\hline Middle tertile & 238 & $162(68.1)$ & 0.91 & $0.76-1.04$ & \\
\hline Upper tertile & 241 & $145(60.2)$ & 0.78 & $0.64-0.92$ & \\
\hline
\end{tabular}

${ }^{1}$ Prevalence ratio. ${ }^{2} p$ value was generated by likelihood ratio test. ${ }^{3}$ Classified based on WHO growth charts. Factors that were not significantly related to acne vulgaris included father's $(p=0.494)$ and mother's $(p=0.081)$ education (less than intermediate school, completed secondary school, completed diploma, completed university degree or above), total number of siblings ( $p=0.690 ; 0-2,3-4,5$ and more), taking supplements in the past 6 months $(p=0.222$; yes/no), physical activity ( $p=0.491$; total time categorized into tertiles), using sunscreen ( $p=0.544$; daily, often, rarely, never), consumption of each food item in the past 3 months (every day, $4-6$ days/ week, $1-3$ days/week, 1 day/week, never): sweets and chocolate $(p=0.815)$, vegetables $(p=0.850)$, whole cream milk $(p=0.185)$, whole-fat cream cheese $(p=0.848)$, low-fat cheese $(p=0.485)$, white cheese $(p=0.235)$, yogurt $(p=0.207)$, ice cream $(p=0.174)$, any other dairy product $(p=0.531)$, nuts $(p=0.370)$, or spicy foods $(p=0.624)$ in addition to measured calcium ( $p=0.781$; categorized into tertiles), protein $(p=0.230$; categorized into tertiles), albumin $(p=0.604 ;<35$ and $\geq 35 \mathrm{~g} / \mathrm{L})$, iron $(p=0.088$; categorized into tertiles), transferrin $(p=0.111$; categorized into tertiles), ferritin ( $p=0.478$; normal $\geq 15 \mathrm{ng} / \mathrm{mL}$ and low $<15 \mathrm{ng} / \mathrm{mL})$, hemoglobin or anemia $(p=0.182$; categorized based on WHO definition of anemia), vitamin $\mathrm{B}_{12}(p=0.326 ;>221,148-221$, and $<148 \mathrm{pmol} / \mathrm{L})$, vitamin $\mathrm{D}(p=0.609 ;<25, \geq 25$ to $<50, \geq 50$ to $<75,75 \mathrm{nmol} / \mathrm{L}$ and more $)$, and parathyroid hormone $(p=0.376$; categorized into tertiles). 
Table 3. Factors associated with clinically examined acne vulgaris among adolescents in Kuwait $(n=714)$ in multivariable logistic regression analysis

\begin{tabular}{|c|c|c|c|c|c|}
\hline Variables & $N$ & Prevalence, $n(\%)$ & PR & $95 \% \mathrm{CI}$ & $p$ value $^{1}$ \\
\hline \multicolumn{6}{|l|}{ Age } \\
\hline 10 years & 301 & $189(61.8)$ & 1.00 & Reference & \multirow[t]{3}{*}{0.003} \\
\hline 12 years & 275 & $192(69.8)$ & 1.17 & $1.06-1.26$ & \\
\hline 13 years & 138 & $101(73.2)$ & 1.19 & $1.06-1.30$ & \\
\hline \multicolumn{6}{|l|}{ Gender } \\
\hline Male & 351 & $210(59.8)$ & 1.00 & Reference & \multirow[t]{2}{*}{0.006} \\
\hline Female & 363 & $269(74.1)$ & 1.16 & $1.05-1.25$ & \\
\hline \multicolumn{6}{|l|}{ Nationality } \\
\hline Kuwaiti & 511 & $368(72.0)$ & 1.00 & Reference & \multirow[t]{2}{*}{$<0.001$} \\
\hline Non-Kuwaiti & 203 & $111(54.7)$ & 0.76 & $0.62-0.90$ & \\
\hline \multicolumn{6}{|c|}{ Body mass index categories } \\
\hline Underweight & 11 & $5(45.4)$ & 0.76 & $0.34-1.18$ & \multirow[t]{4}{*}{0.026} \\
\hline Normal weight & 313 & $194(62.0)$ & 1.00 & Reference & \\
\hline Overweight & 158 & $116(73.4)$ & 1.15 & $1.02-1.26$ & \\
\hline Obesity & 232 & $164(70.7)$ & 1.14 & $1.02-1.23$ & \\
\hline
\end{tabular}

${ }^{1} p$ values were generated using likelihood ratio test.

41.1-48.6\%) self-reported AV. This was significantly higher among females compared to males (56.2 vs. 33.0\%, respectively; $p<0.001)$. By clinical examination, AV was found in 479 (67.1\%; 95\% CI 63.5-70.5\%) participants. The prevalence of clinically assessed AV was significantly higher among females compared to males ( 74.1 vs. $59.8 \%$, respectively; $p<0.001)$. The prevalence of self-reported $\mathrm{AV}$ and clinically assessed AV significantly increased with age (chi-squared for trend $p=0.004$ and $p=0.001$, respectively). As per the GAGS, there were no severe cases of AV; all cases were either mild or moderate.

Factors that showed significant association with clinically examined AV in univariable analysis are shown in Table 2. Age, gender, nationality, BMI categories, consumption of fruits, fried food, or low-fat milk in the past 3 months, in addition to transferrin saturation were all significantly associated with AV in univariable analysis. The median (interquartile range) of $Z$ score for BMI-forage was $1.31(0.33-2.4)$ and $0.82(-0.27$ to 2.17$)$ among those with and without AV $(p=0.002)$. Factors that were not significantly associated with AV included the level of education of the father $(p=0.494)$ and the mother $(p=$ $0.081)$, total number of siblings $(p=0.690)$, taking vitamin supplement in the past 6 months $(p=0.222)$, physical activity $(p=0.491)$, using sunscreen $(p=0.544)$, consumption of each food item: sweets and chocolate ( $p=$ $0.815)$, vegetables $(p=0.850)$, whole cream milk ( $p=$ $0.185)$, whole-fat cream cheese $(p=0.848)$, low-fat cheese $(p=0.485)$, white cheese $(p=0.235)$, yogurt $(p=0.207)$, ice cream $(p=0.174)$, any other dairy products $(p=0.531)$, nuts $(p=0.370)$, and spicy food $(p=0.624)$, in addition to measured calcium $(p=0.781)$, protein $(p=0.230)$, albumin $(p=0.604)$, iron $(p=0.088)$, transferrin $(p=0.111)$, ferritin $(p=0.478)$, hemoglobin or anemia $(p=0.182)$, vitamin $\mathrm{B}_{12}(p=0.326)$, vitamin $\mathrm{D}(p=0.609)$, and $\mathrm{PTH}$ $(p=0.376)$. In multivariable analysis, only age, gender, nationality, and BMI categories were significantly associated with clinically assessed AV (Table 3 ). In this analysis, females compared to males and obese/overweight compared to adolescents with normal weight were more likely to have AV.

\section{Discussion}

This study aimed to estimate the prevalence of $\mathrm{AV}$ and investigate the factors associated with AV among school adolescents in Kuwait. To the best of our knowledge, this is the first study in Kuwait to estimate the prevalence of AV in a population-based study. The prevalence of selfreported AV among school adolescents was found to be $44.8 \%$, which is similar to that reported from Poland (42.2\% based on online survey among adolescents 15-24 years old) but substantially less than the self-reported prevalence of $\mathrm{AV}$ in several other settings such as the Czech and Slovak Republic (73.5\%) [8] and Serbia (51.8\% among students with mean age 16.5 years) [14]. The prevalence of AV by clinical examination in our study was 
67.1\%, which was lower than that reported from Nigeria, where $97.1 \%$ of 17 - to 19 -year-old students were diagnosed with AV [7] but higher than the prevalence in China (46.8\% of adolescents aged 19 years) [15] and similar to that reported from Malaysia (67.5\% of secondary school students) [16]. A much higher prevalence of AV by clinical examination has been reported from other settings such as Brazil ( $96 \%$ of adolescents aged $11-17$ years) [2] and Iran (93.3\% of adolescents aged 12-20 years) [9]. The prevalence of AV (self-reported or by clinical examination) varies considerably between different settings. It is not clear whether this variation reflects genuine differences in the prevalence of AV between geographical settings or is due to different methodological approaches.

We reported higher prevalence of AV by clinical examination compared to self-reported AV. This was similar to studies in other settings [17], where the prevalence of self-reported AV was lower compared to AV by clinical assessment. It is well known that patients' perception of $\mathrm{AV}$ does not always correspond to the diagnosis made in clinical examination [2]. It is possible that adolescents, particularly those with mild forms of AV, may prefer not to declare themselves as having $\mathrm{AV}$, particularly when they compare their case with others who have more severe forms of $\mathrm{AV}$. Previous studies also suggest that the majority of adolescents consider AV not as a disease but as a normal event during adolescence [5].

The prevalence of clinically assessed AV and self-reported AV were significantly higher in females compared to males in our setting, even after stratification by age, which is similar to that reported from Taiwan [18]. Many other studies reported males to have significantly higher prevalence of AV compared to females [15, 16]. Several plausible biological factors may explain the higher prevalence of AV among males compared to females, including the substantial increase in androgens that occurs after puberty. One of the plausible explanations for our finding is that both clinicians who examined AV among adolescents were females; it is possible that they felt more comfortable with female participants, hence searched for AV more thoroughly in the back and chest of females compared to the back and chest of males due to gender discordance. However, while this can explain the difference in the prevalence of $\mathrm{AV}$ by clinical examination, it cannot explain the difference in the prevalence of self-reported AV. Differences in psychological triggers of AV could explain the higher prevalence of AV (self-reported and clinically diagnosed AV) among females compared to males. Polycystic ovary syndrome could also be responsible for some AV cases among females. Moreover, although we

Predictors of Acne Vulgaris among

Adolescents have roughly equal numbers of females and males in this study, our finding could also be attributed to bias if males with AV were more likely to be absent from schools on the days of data collection compared to females with AV.

With respect to the factors associated with clinically assessed AV, the only modifiable factor that was significantly associated with AV in multivariable analysis was obesity. Obese/overweight adolescents were more likely to have AV compared to adolescents with normal weight in univariable and multivariable analysis (Table 2, 3). In Taiwan, the mean BMI of school children (aged 6-11 years) was higher among participants with AV compared to those without AV [18]. However, this comparison is not meaningful because BMI has to be linked to growth charts in this age group. In Italy, higher risk of AV was reported among those with higher BMI [19], but in another study in Sydney, no association was found between increased BMI and AV [20]. In Norway, overweight/obesity was associated with AV in girls but not in boys [21]. Obesity has been suggested to be accompanied by peripheral hyperandrogenism, which might be associated with increased production of sebum leading to AV [19]. Finally, it is possible that the association between obesity/ overweight and $\mathrm{AV}$ is due to residual confounding by diet, which is difficult to measure, particularly over a long period of time.

Over several decades, attempts to link diet to AV have so far produced inconsistent results. Foods that have been implicated in the development and/or severity of AV include milk and dairy products, chocolate, food with high glycemic index, dietary fiber intake, fish, and seafood [22]. Most studies, however, attempted to investigate the impact of diet on the severity of AV rather than the occurrence of $\mathrm{AV}$. There is growing evidence that supports the beneficial effect of low glycemic index diet for acne patients [23]. In our study, none of the food items was associated with clinically assessed AV in multivariable analysis. Frequency of consumption of fruits was negatively associated with AV in univariable analysis but lost statistical significance in multivariable analysis. In a crude comparison between 453 cases of AV and 250 controls without $\mathrm{AV}$, a vegan diet was more common among the control group [20]. Fruit consumption is usually associated with less consumption of fruit-flavored sugary drinks, which is very common among children in our setting. We did not find an association between clinically assessed AV and frequency of consumption of sweets and chocolate, neither in univariable nor multivariable analysis. Neither consumption of milk nor of any other dairy product was associated with AV in multivariable analysis, 
but consumption of low-fat milk was associated with AV in univariable analysis. Several observational studies have shown the association between AV and consumption of milk or low-fat milk, as reviewed by Bronsnick et al. [23]. Consumption of high-glycemic food items and milk are hypothesized to increase the levels of insulin and serum insulin growth factor-1, which promote comedogenesis, sebaceous lipogenesis, follicular inflammation, and androgenic stimulation, which hence leads to severe AV [24].

The link between AV and vitamins has attracted great attention. Previously, we have demonstrated that levels of vitamin D are not related to AV [11]. There are several reports of attack of acne or acne-like disease after large doses of vitamin $B_{12}$ supplementation [25]. It has been hypothesized that vitamin $\mathrm{B}_{12}$ modulates the activities of the skin microbiota and contributes to AV pathogenesis [26]. On the other hand, reports have linked vitamin $B_{12}$ deficiency to the treatment of AV by isotretinoin [27]. In our data, there was no association between levels of vita$\min B_{12}$ and $A V$ in univariable nor multivariable analysis. Other laboratory measures such as folate, hemoglobin, ferritin, total protein, and albumin showed no significant difference between those with and without AV.

One of the strengths of this study is collecting the data on risk factors and potential confounders from both parents and adolescents. Missing values were minimal and are unlikely to affect our findings. However, the study has several limitations including the intrinsic weakness of cross-sectional design, which does not allow us to ascertain the temporal relationship between presumed risk factors and AV. For example, it is not clear if obesity occurred before or after AV. For logistical reasons, we collected data on 714 participants out of 1,416 adolescents recruited in the main project. Table 1 shows that the char- acteristics of the 2 study groups are similar. Furthermore, although there is no strong evidence from the literature that AV is associated with school absenteeism, one could speculate that those most severely affected by AV were missed because they are more likely to be absent from the schools and hence less likely to participate in the study. If this happened, it would underestimate the prevalence of $\mathrm{AV}$ and attenuate the association of AV with some risk factors. Finally, we used GAGS to measure the severity of $A V$, which may explain the absence of severe cases in our study.

\section{Conclusion}

The prevalence of self-reported AV was estimated to be $44.8 \%$, which seems to be lower than that reported from several other settings, while the prevalence of AV by clinical assessment was approximately $67.1 \%$, which resembles that reported in many other countries. BMI was the only modifiable risk factor associated with AV in multivariable analysis; none of the food items studied was related to the presence or absence of AV in multivariable analysis.

\section{Statement of Ethics}

The study was approved by the Ethics Committee at the Ministry of Health in Kuwait (No. 2015/248) and written parental informed consent was obtained for each study participant.

\section{Disclosure Statement} close.

\section{References}

1 Tan JK, Bhate K. A global perspective on the epidemiology of acne. Br J Dermatol. 2015 Jul; 172 Suppl 1:3-12.

2 Bagatin E, Timpano DL, Guadanhim LR, Nogueira VM, Terzian LR, Steiner D, et al. Acne vulgaris: prevalence and clinical forms in adolescents from São Paulo, Brazil. An Bras Dermatol. 2014 May-Jun;89(3):428-35.

3 Bhate K, Williams HC. Epidemiology of acne vulgaris. Br J Dermatol. 2013 Mar;168(3): $474-85$.

4 Karimkhani C, Dellavalle RP, Coffeng LE, Flohr C, Hay RJ, Langan SM, et al. Global Skin Disease Morbidity and Mortality: An Update From the Global Burden of Disease Study 2013. JAMA Dermatol. 2017 May;153(5):406-12.
5 Uslu G, Sendur N, Uslu M, Savk E, Karaman G, Eskin M. Acne: prevalence, perceptions and effects on psychological health among adolescents in Aydin, Turkey. J Eur Acad Dermatol Venereol. 2008 Apr;22(4): 462-9.

6 Lukaviciute L, Navickas P, Navickas A, Grigaitiene J, Ganceviciene R, Zouboulis CC. Quality of life, anxiety prevalence, depression symptomatology and suicidal ideation among acne patients in Lithuania. J Eur Acad Dermatol Venereol. 2017 Nov;31(11):1900-6.

7 Yahya $\mathrm{H}$. Acne vulgaris in Nigerian adolescents-prevalence, severity, beliefs, perceptions, and practices. Int J Dermatol. 2009 May;48(5):498-505.
8 Wolkenstein P, Machovcová A, Szepietowski JC, Tennstedt D, Veraldi S, Delarue A. Acne prevalence and associations with lifestyle: a cross-sectional online survey of adolescents/ young adults in 7 European countries. J Eur Acad Dermatol Venereol. 2018 Feb;32(2): 298-306.

9 Ghodsi SZ, Orawa H, Zouboulis CC. Prevalence, severity, and severity risk factors of acne in high school pupils: a communitybased study. J Invest Dermatol. 2009 Sep; 129(9):2136-41.

10 Nanda A, Al-Hasawi F, Alsaleh QA. A prospective survey of pediatric dermatology clinic patients in Kuwait: an analysis of 10,000 cases. Pediatr Dermatol. 1999 Jan-Feb;16(1):6-11. 
11 Al-Taiar A, AlKhabbaz M, Rahman A, Al-Sabah R, Shaban L, Akhtar S. Plasma 25-Hydroxy Vitamin D is not Associated with Acne Vulgaris. Nutrients. 2018 Oct;10(10):10.

12 Al-Taiar A, Rahman A, Al-Sabah R, Shaban L, Al-Harbi A. Vitamin D status among adolescents in Kuwait: a cross-sectional study. BMJ Open. 2018 Aug;8(7):e021401.

13 Doshi A, Zaheer A, Stiller MJ. A comparison of current acne grading systems and proposal of a novel system. Int J Dermatol. 1997 Jun; 36(6):416-8.

14 Perić J, Maksimović N, Janković J, Mijović B, Reljić V, Janković S. Prevalence and quality of life in high school pupils with acne in Serbia. Vojnosanit Pregl. 2013 Oct;70(10):935-9.

15 Shen Y, Wang T, Zhou C, Wang X, Ding X, Tian S, et al. Prevalence of acne vulgaris in Chinese adolescents and adults: a community-based study of 17,345 subjects in six cities. Acta Derm Venereol. 2012 Jan;92(1):40-4.

16 Hanisah A, Omar K, Shah SA. Prevalence of acne and its impact on the quality of life in school-aged adolescents in Malaysia. J Prim Health Care. 2009 Mar;1(1):20-5.
17 Ogedegbe EE, Henshaw EB. Severity and impact of acne vulgaris on the quality of life of adolescents in Nigeria. Clin Cosmet Investig Dermatol. 2014 Dec;7:329-34.

18 Tsai MC, Chen W, Cheng YW, Wang CY, Chen GY, Hsu TJ. Higher body mass index is a significant risk factor for acne formation in schoolchildren. Eur J Dermatol. 2006 MayJun;16(3):251-3.

19 Di Landro A, Cazzaniga S, Parazzini F, Ingordo V, Cusano F, Atzori L, et al.; GISED Acne Study Group. Family history, body mass index, selected dietary factors, menstrual history, and risk of moderate to severe acne in adolescents and young adults. J Am Acad Dermatol. 2012 Dec;67(6):1129-35.

20 Stewart TJ, Bazergy C. Hormonal and dietary factors in acne vulgaris versus controls. Dermatoendocrinol. 2018 Feb;10(1):e1442160.

21 Halvorsen JA, Vleugels RA, Bjertness E, Lien L. A population-based study of acne and body mass index in adolescents. Arch Dermatol. 2012 Jan;148(1):131-2
22 Kucharska A, Szmurło A, Sińska B. Significance of diet in treated and untreated acne vulgaris. Postepy Dermatol Alergol. 2016 Apr;33(2):81-6.

23 Bronsnick T, Murzaku EC, Rao BK. Diet in dermatology: Part I. Atopic dermatitis, acne, and nonmelanoma skin cancer. J Am Acad Dermatol. 2014 Dec;71(6):1039.e1-1039.e12.

24 Melnik BC. Evidence for acne-promoting effects of milk and other insulinotropic dairy products. Nestle Nutr Workshop Ser Pediatr Program. 2011;67:131-45.

25 Sherertz EF. Acneiform eruption due to "megadose" vitamins B6 and B12. Cutis. 1991 Aug;48(2):119-20.

26 Kang D, Shi B, Erfe MC, Craft N, Li H. Vitamin B12 modulates the transcriptome of the skin microbiota in acne pathogenesis. Sci Transl Med. 2015 Jun;7(293):293ra103.

27 Karadag AS, Tutal E, Ertugrul DT, Akin KO. Effect of isotretinoin treatment on plasma holotranscobalamin, vitamin B12, folic acid, and homocysteine levels: non-controlled study. Int J Dermatol. 2011 Dec;50(12):1564-9. 